\title{
The effects of iPod and text-messaging use on driver distraction: a bio-behavioral analysis
}

\author{
M. Mouloua*a; A. Ahern ${ }^{\text {a }}$; A. Quevedo ${ }^{\text {d}}$; D. Jaramillo $;$ E. Rinalducci ${ }^{\text {a }}$ J. Smither ${ }^{\text {a }}$; P. Alberti ${ }^{\text {b }}$; \\ and C. Brill ${ }^{\mathrm{c}}$ \\ ${ }^{a}$ Psychology Department, University of Central Florida, 4000 Central Florida Blvd. Bldg. 99 Rm 326 \\ Orlando, FL 32816-1390, USA \\ ${ }^{\mathrm{b}}$ Department of Technology and Human Sciences, Université de Technologie de Compiegne, France \\ ' Department of Psychology, Old Dominion University, Norfolk, VA, USA \\ d Optimal Electro Physiology, Orlando, FL, USA
}

\begin{abstract}
This study was designed to empirically examine the effects of iPod device and text-messaging activities on driver distraction. Sixty participants were asked to perform a driving simulation task while searching for songs using an iPod device or text messaging. Driving errors as measured by lane deviations were recorded and analyzed as a function of the distracters. Physiological measures (EEG) were also recorded during the driving phases in order to measure participant levels of cortical arousal. It was hypothesized that iPod use and text messaging would result in a profound effect on driving ability. The results showed a significant effect of iPod use and text-messaging on driving performance. Increased numbers of driving errors were recorded during the iPod and text-messaging phases than the pre- and post-allocation phases. Higher levels of Theta activity were also observed during the iPod and Text-messaging phase than the pre- and post-allocation phases. Implications for in-vehicle systems design, training, and safety are also discussed.
\end{abstract}

Keywords: Text-Messaging; iPod Use; Driver Distraction; In-Vehicle devices

\section{Introduction}

Driver distraction with different in-vehicle devices has been widely researched for some time. The main conclusion that has arisen from such studies is that dual-tasking while driving an automobile leads to impaired performance [1-2, 4-6]. Although some people may rely on their perceived ability to multitask, it has been found that when engaged in secondary activities while driving there is competition for cognitive resources, amongst the different tasks, that leads to impaired driver performance $[2,4,6]$. Results of a study conducted using functional magnetic resonance imaging (fMRI), found that spatial processing was reduced by $37 \%$ in participants who were actively listening to sentences while engaged in simulated driving [4]. This same principle applies to vision as well, where as a shared resource it must be allocated to all different activities involved with each task. The fact that there is a limited capacity of the visual resource suggests the reason for the impaired driving $[2,6]$.
Another study tested driver scanning behaviors while using hands-free in-vehicle technologies. The participants showed marked changes in their visual allocation once engaged in a complex secondary task, spending less time looking at the mirrors and periphery and only looking ahead and to the telematic device [2]. On the same hand, the SEEV visual scanning model was tested to determine its usefulness in predicting driver scanning behaviors when responding to road hazards. This model may aid in understanding visual attention allocation in different driving circumstances so as to better design future technology [3]. Much of the research has lead scientists to conclude that although some in-vehicle technologies (e.g., collision warning systems) may be beneficial to the driver, some others (e.g., radio) may largely contribute to crashes and other impaired driving behaviors. Thus, suggesting that both positive and negative consequences of technology use must be taken into account when designing new forms of technology so as to enhance the positive and minimize the negative $[2,5]$. Music players, such as

Corresponding author. E-mail: Mustapha.Mouloua@ucf.edu. Tel: 407-823-2910.

1051-9815/12/\$27.50 (C) 2012 - IOS Press and the authors. All rights reserved 
iPods, have become increasingly popular recently. However, few studies have been conducted to assess the distraction implications of such devices [6]. The unique feature about this study is that by implementing EEG we can quantitatively measure the physiological features underlying the observed impaired driving behavior. The use of a bio-behavioral assessment method to determine the effects of these distraction factors on our ability to drive safely is a unique aspect of our experimental approach.

\section{Experiment one}

\section{Method}

Experiment One was designed to empirically examine the effects of iPod devices on driver distraction. It was hypothesized that during the iPod trial the participants would display higher levels of arousal and a prevalence of the theta frequency (4-7 $\mathrm{Hz}$ ), associated with distractibility, as well as an impaired driving performance indicated by a higher incidence of driving errors.

\subsection{Participants and task procedures}

Thirty participants were required to perform a driving simulation task while searching for songs using an iPod device. Driving errors as measured by lane deviations were recorded and analyzed as a function of the distracter. Physiological measures (EEG) were also recorded during the driving phases to quantitatively measure the participant's level of cortical arousal.

\subsection{Results of experiment one}

The results of the EEG showed a significant effect of iPod use on theta activity $F(2,28)=13.26, p<0.001$. This indicates that the device caused increased inattention, which is represented by higher Theta (Mean=19.91) during the iPod use than the pre-iPod (Mean=17.24) or post-iPod (Mean=17.02) use. Also, the results showed a significant effect of iPod use on driving errors, $F(2,28)=37.24, p<0.001$. This indicates that more driving errors occurred during the iPod (Mean=6.93) than the pre-iPod $($ Mean=3.27) or post-iPod (Mean=3.40) use.

\section{Experiment Two}

\section{Method}

Experiment Two was designed to empirically examine the effects of text-messaging on driver distraction. It was hypothesized that text-messaging would affect driving ability and the level of cortical arousal.

\subsection{Participants and task procedures}

Thirty participants were required to perform a driving simulation task while text-messaging using a cellular phone device. Driving errors as measured by lane deviations were also recorded and analyzed as a function of the distracter as in Experiment 1.

\subsection{Results of experiment two}

The results of the EEG showed a significant effect of text messaging use on theta activity $F(2,26)=15.28, p$ $<0.001$. This indicates that the use of cellular phones while driving caused increased inattention levels, which is represented by higher Theta $(M=21.71)$ during the text messaging phase than during the pre-textmessaging $(M=15.19)$ or post-texting $\quad(M=20.01)$ phases. Also, the results showed a significant effect of text messaging on driving errors, $F(2,26)=42.16, p<$ 0.001 . This indicates that more driving errors occurred during the text messaging phase $(M=10.71)$ than the pre-text messaging $(M=5.51)$ or post-text-messaging $(M=4.96)$ phases

\section{Conclusions}

The present findings indicate that engaging in distracting activities such as searching for songs in an iPod device or text-messaging are both cognitively demanding tasks which can lead to impaired driver performance. This is shown by the increased number of driving errors committed during the iPod and textmessaging trials. These results are consistent with previous studies that have concluded that dual-tasking while driving and automobile leads to impaired driving performance [1-2, 4-6]. In addition, it is also evident that there was an increased cortical arousal and prevalence of theta during the iPod and text-messaging trials. 


\section{References}

[1] N. Dibben \& V. Williamson (2007). An exploratory survey of in-vehicle music listening. Psychology of Music, 35(4): 571-589.

[2] J. L. Harbluk, J. I. Noy, P. L. Trbovich, \& M. Eizenman. (2007). An on-road assessment of cognitive distraction: Impacts on driver's visual behavior and braking performance. Accident Analysis \& Prevention, 39, Issue 2, 372-379.

[3] W. J. Horrey, C. D. Wickens, \& K. P. Consalus. (2006)

Modeling driver's visual attention allocation while interacting with in-vehicle technologies. Journal of Experimental Psychology: Applied, 12(2), 67-78.

[4] M. A. Just, T. A. Keller, \& J. Cynkar. (n. y.). A decrease in brain activation associated with driving when listening to someone speak. Brain Research, 1205, 70-80.

[5] J. D. Lee. (2007). Technology and teen drivers. Journal of Safety Research, 38(2), 203-213.

[6] D. D. Salvucci, D. Markley, M. Zuber, \& D. P. Brumby. (2007). iPod distraction: Effects of portable music-player use on driver performance. Task and Attention, 243-250. 